\title{
Three-body problem in heteronuclear mixtures with resonant interspecies interaction
}

\author{
K. Helfrich, ${ }^{1}$ H.-W. Hammer, ${ }^{1}$ and D. S. Petrov ${ }^{2,3}$ \\ ${ }^{1}$ Helmholtz-Institut für Strahlen- und Kernphysik (Theorie) \\ and Bethe Center for Theoretical Physics, Universität Bonn, 53115 Bonn, Germany \\ ${ }^{2}$ Laboratoire Physique Théorique et Modèles Statistique, \\ Université Paris Sud, CNRS, 91405 Orsay, France \\ ${ }^{3}$ Russian Research Center Kurchatov Institute, Kurchatov Square, 123182 Moscow, Russia
}

(Dated: November 14, 2018)

\begin{abstract}
We use the zero-range approximation to study a system of two identical bosons interacting resonantly with a third particle. The method is derived from effective field theory. It reduces the three-body problem to an integral equation which we then solve numerically. We also develop an alternative approach which gives analytic solutions of the integral equation in coordinate representation in the limit of vanishing total energy. The atom-dimer scattering length, the rates of atom-dimer relaxation, and three-body recombination to shallow and to deep molecular states are calculated either analytically or numerically with a well-controlled accuracy for various energies as functions of the mass ratio, scattering length, and three-body parameter. We discuss in detail the relative positions of the recombination loss peaks, which in the universal limit depend only on the mass ratio. Our results have implications for ongoing and future experiments on Bose-Bose and Bose-Fermi atomic mixtures.
\end{abstract}

PACS numbers: 34.50.-s, 67.85.Pq

\section{INTRODUCTION}

The universal properties of particles with resonant short-range interactions are a subject of intense research. Such systems are characterized by a large scattering length and display universal phenomena associated with a discrete scaling symmetry [1, 2]. For identical bosons, Efimov found that there are infinitely many trimer states with an accumulation point at the scattering threshold when the $s$-wave scattering length $a$ is tuned to the unitary limit $1 / a=0[3]$ :

$$
B_{t}^{(n)}=\left(e^{-2 \pi / s_{0}}\right)^{n-n_{*}} \hbar^{2} \kappa_{*}^{2} / m,
$$

where $m$ is the mass of the particles, $s_{0} \approx 1.00624$, and $\kappa_{*}$ is the binding wavenumber of the branch of Efimov states labeled by $n_{*}$. The geometric spectrum in (1) is the signature of a discrete scaling symmetry with scaling factor $e^{\pi / s_{0}} \approx 22.7$. For a finite scattering length larger than the range of the interaction, the universal properties persist but there is only a finite number of Efimov states.

Ultracold atoms are an ideal tool to study such phenomena since the scattering length can be tuned experimentally using Feshbach resonances. Efimov trimers in ultracold atomic gases can be observed via their signature in three-body recombination rates 448 . Kraemer et al. provided the first evidence for Efimov trimers in an ultracold gas of ${ }^{133} \mathrm{Cs}$ atoms by observing the resonant enhancement of three-body recombination caused by the trimers 9 . In a subsequent experiment with a mixture of ${ }^{133} \mathrm{Cs}$ atoms and dimers, Knoop et al. observed a resonance in the loss of atoms and dimers 10 which can be explained by an Efimov trimer crossing the atom-dimer threshold [11.

Several recent experiments have also obtained evidence of Efimov physics with other bosonic atoms.
Zaccanti et al. measured the three-body recombination rate and the atom-dimer loss rate in an ultracold gas of ${ }^{39} \mathrm{~K}$ atoms 12 . They observed loss features at large positive and negative values of the scattering length, positions of which agree with the discrete scaling symmetry. Gross et al. measured the three-body recombination rate in an ultracold system of ${ }^{7} \mathrm{Li}$ atoms [13. They observed a three-atom loss resonance and a three-body recombination minimum in the same universal region on different sides of a Feshbach resonance. Their positions are consistent with the universal predictions with discrete scaling factor of 22.7. Using ultracold ${ }^{7} \mathrm{Li}$ atoms as well, Pollack et al. 14 observed 11 three- and four-body loss features in the inelastic loss spectrum. Their relative locations on either side agree well with the universal theory, while a systematic deviation from universality appears when comparing features across the resonance. The origin of this deviation is not understood. Barontini et al. 15. investigated the Bose-Bose mixture ${ }^{41} \mathrm{~K}-{ }^{87} \mathrm{Rb}$ and found three resonance positions in the three-body loss. The two features for negative scattering length were attributed to the two possible Efimov trimers of the system, K-Rb-Rb and $\mathrm{K}-\mathrm{K}-\mathrm{Rb}$, hitting the three-atom threshold.

The Efimov effect can also occur for fermionic atoms with at least three spin states. The first experimental studies of many-body systems of ${ }^{6} \mathrm{Li}$ atoms in the three lowest hyperfine states were recently carried out by Ottenstein et al. [16] and Huckans et al. [17. Theoretical calculations of the three-body recombination rate supported the interpretation that the narrow loss feature arises from an Efimov trimer crossing the three-atom threshold [18 21]. Very recently, another narrow loss feature was discovered in the region of much higher magnetic fields by Williams et al. 22] and by Jochim and co-workers 23]. In this region, the scattering length is 
much larger and several recombination features have been predicted using the universal theory [24].

In this paper, we focus on heteronuclear systems with two species of atoms where only the interspecies scattering length is large. For comparable masses the scaling factor is quite large (for equal masses $e^{\pi / s_{0}} \approx 1986.1$ ) as we now have only two resonant interactions out of three. However, in the case of two heavy atoms and one light atom, this factor can become significantly smaller than the value 22.7 for identical bosons [1, 25 27, which should stimulate experimental investigation of the discrete scaling invariance. Relaxation and recombination losses near an interspecies resonance have recently been investigated in mixtures of rubidium and potassium. The Bose-Fermi combination ${ }^{87} \mathrm{Rb}^{40} \mathrm{~K}$ has been studied at the Joint Institute for Laboratory Astrophysics (JILA) 28] and measurements on the Bose-Bose mixture ${ }^{41} \mathrm{~K}-{ }^{87} \mathrm{Rb}$ have been carried out in Florence [15]. We apply our theory to these and other mixtures of interest for ongoing and planned experiments.

\section{METHOD}

In this section, we set up the effective field theory method which provides a convenient implementation of the universal theory for large scattering length. We set $\hbar=1$ but restore the dimensions in our expressions for the recombination rate constants. We consider a system of one boson or fermion of mass $m_{1}$ (species 1) and two identical bosons of mass $m_{2}$ (species 2). We assume the interspecies interaction to be resonant and characterized by the $s$-wave scattering length $a \gg \ell_{v d W}$, where $\ell_{v d W}$ is the van der Waals range of the potential. Nonresonant intraspecies interaction will be neglected. If species 1 is also bosonic and weakly interacting, all the forthcoming results directly apply to the other possible (interacting) triple by simply exchanging the labels 1 and 2. We therefore include only the interaction between the atoms of species 2 and the dimers. Hence, our effective Lagrangian reads

$$
\begin{aligned}
\mathcal{L}= & \psi_{1}^{\dagger}\left(i \partial_{t}+\frac{\nabla^{2}}{2 m_{1}}\right) \psi_{1}+\psi_{2}^{\dagger}\left(i \partial_{t}+\frac{\nabla^{2}}{2 m_{2}}\right) \psi_{2}+g_{2} d^{\dagger} d \\
& -g_{2}\left(d^{\dagger} \psi_{1} \psi_{2}+\psi_{1}^{\dagger} \psi_{2}^{\dagger} d\right)-\frac{g_{3}}{4} d^{\dagger} d \psi_{2}^{\dagger} \psi_{2}+\cdots, \quad(2)
\end{aligned}
$$

where the dots represent higher-order derivative interactions, and $g_{2}$ and $g_{3}$ are the bare two- and three-body coupling constants.

From the Lagrangian (2), we can deduce Feynman rules and obtain the full dimer propagator and the threebody integral equation (see Ref. [1] for details on the derivation). For the full dimer propagator we get

$$
D\left(P_{0}, \mathbf{P}\right)=\frac{2 \pi}{g_{2}^{2} \mu}\left[\frac{1}{a}-\sqrt{-2 \mu\left(P_{0}-\frac{P^{2}}{2 M}\right)-i \epsilon}\right]^{-1},
$$

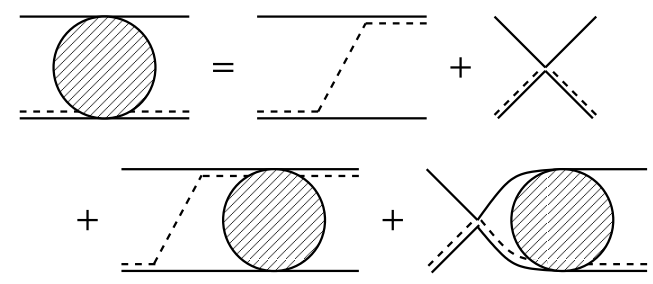

FIG. 1: Integral equation for the atom-dimer scattering amplitude $\mathcal{A}$. Solid (dashed) lines denote atom species 2 (1). Mixed double lines denote the full dimer propagator.

where $P=|\mathbf{P}|, \mu=m_{1} m_{2} /\left(m_{1}+m_{2}\right)$ is the reduced mass, $M=m_{1}+m_{2}$ is the mass of the dimer, and the limit of $\epsilon \rightarrow+0$ is understood. The dimer wave function renormalization is given by $Z_{D}^{-1}=g_{2}^{2} a \mu^{2} /(2 \pi)$.

The scattering between a dimer and an atom is described by the integral equation shown in Fig. 1. Using the Feynman rules derived from Eq. (2) and projecting on relative $s$-waves, we have

$$
\begin{aligned}
\mathcal{A}(p, k ; E)= & \frac{2 \pi m_{1}}{a \mu^{2}}\left[K(p, k)-\frac{g_{3}}{4 m_{1} g_{2}^{2}}\right] \\
& +\frac{m_{1}}{\pi \mu} \int_{0}^{\Lambda} d q q^{2}\left[K(p, q)-\frac{g_{3}}{4 m_{1} g_{2}^{2}}\right] \\
& \times \frac{\mathcal{A}(q, k ; E)}{-\frac{1}{a}+\sqrt{-2 \mu\left(E-\frac{q^{2}}{2 \mu_{A D}}\right)-i \epsilon}}
\end{aligned}
$$

where $\mu_{A D}=m_{2}\left(m_{1}+m_{2}\right) /\left(2 m_{2}+m_{1}\right)$ is the reduced mass of an atom and a dimer, the relative momenta of the incoming and outgoing atom-dimer pair are denoted by $p$ and $k$, respectively; and $E$ is the total energy. The contribution of the $s$-wave projected one-atom exchange is given by

$$
K(p, q)=\frac{1}{2 p q} \ln \left[\frac{p^{2}+q^{2}+2 p q \frac{\mu}{m_{1}}-2 \mu E-i \epsilon}{p^{2}+q^{2}-2 p q \frac{\mu}{m_{1}}-2 \mu E-i \epsilon}\right],
$$

and the contribution of the three-body coupling $g_{3}$ can be written as

$$
\frac{g_{3}}{4 m_{1} g_{2}^{2}}=-\frac{H(\Lambda)}{\Lambda^{2}}
$$

where $H(\Lambda)$ is a dimensionless log-periodic function of the cutoff $\Lambda$, which depends on a three-body parameter $\Lambda_{*}$ [29]. The mass-ratio dependence of the discrete scaling factor $\exp \left(\pi / s_{0}\right)$ follows from the equation for $s_{0}$ :

$$
s_{0} \cosh \left(\pi s_{0} / 2\right)-2 \sinh \left(\phi s_{0}\right) / \sin (2 \phi)=0,
$$

where we introduce the parameter

$$
\phi=\arcsin [1 /(1+\delta)]
$$


and the notation $\delta=m_{1} / m_{2}$. For particles of equal mass, the solution of Eq. (7) is $s_{0} \approx 0.4137$ leading to the scaling factor $\exp \left(\pi / s_{0}\right) \approx 1986.1$. Because of the log-periodicity of $H(\Lambda)$ one can always find a value of the cutoff $\Lambda$ with $H=0$. In practice, one can therefore simply omit the three-body coupling in the leading-order calculations and use the cutoff $\Lambda$ as a three-body parameter [30. We use this strategy in the following. For fixed $\delta$, the values of $\Lambda$ and $\Lambda_{*}$ are related by a multiplicative constant.

The scattering amplitude $\mathcal{A}$ has simple poles at the three-body bound-state energies $E=-B_{t}<0$. The energies can be obtained from the solution of the following homogeneous integral equation for the bound-state amplitude $\mathcal{B}$ :

$$
\mathcal{B}\left(p ; B_{t}\right)=\frac{m_{1}}{\pi \mu} \int_{0}^{\Lambda} \frac{d q q^{2} K(p, q) \mathcal{B}\left(q ; B_{t}\right)}{-\frac{1}{a}+\sqrt{2 \mu\left(B_{t}+\frac{q^{2}}{2 \mu_{A D}}\right)}},
$$

which has nontrivial solutions only for three-body binding energies $B_{t}>0$. In the following, we use Eqs. (4) and $(9)$ to describe three-body properties of heteronuclear mixtures.

\section{NUMERICAL RESULTS}

Few-body loss phenomena offer a unique view on scattering processes in ultracold quantum gases. In particular, an enhancement of the loss rate can be an evidence of a few-body resonance. The universal theory predicts the relative positions of such resonances as a function of the scattering length. The universality can thus be tested experimentally by measuring the lifetime of a cold atomic gas as a function of $a$. Ideally, in order to see the universal scaling, one needs to detect more than one resonance in a single universal region, that is, a region where the three-body parameter can be assumed constant. This is believed to happen in a narrow vicinity of a Feshbach resonance, where large variations of $a$ are accompanied by (assumed) much weaker variations of the three-body parameter. We now discuss three-body loss resonances in a heteronuclear mixture as predicted by the universal theory.

\section{A. Resonance positions}

The mechanism of three-body losses and its relation to the positions of Efimov levels in the heteronuclear case are qualitatively the same as for three identical bosons. The scattering-length dependence of the energy of a generic trimer is illustrated in Fig. 2, On the negative side of a Feshbach resonance the trimer hits the three-body scattering threshold at $a=a_{-}<0$, which leads to an enhanced probability of finding three atoms at distances of the order of $|a|$. Such atoms can

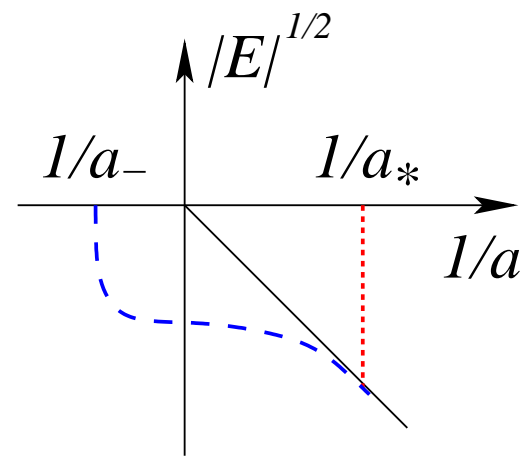

FIG. 2: (Color online) Dependence of the trimer energy on the inverse scattering length $1 / a$ in arbitrary units (dashed line). The parameters $a_{-}$and $a_{*}$ specify where the trimer state hits the three-atom and atom-dimer thresholds, respectively.

then approach each other to distances of the order of $\ell_{v d W}$ and recombine into a deeply bound dimer and a residual atom. The released binding energy [of order $\left.\hbar^{2} /\left(2 \mu \ell_{v d W}^{2}\right)\right]$ transforms into the kinetic energy of the recombination products, which hence leave the trap. On the positive side of the Feshbach resonance there exists a weakly bound (shallow) dimer state with binding energy $B_{d}=\hbar^{2} /\left(2 \mu a^{2}\right)$. This formula taken with minus sign determines the atom-dimer threshold (solid line in Fig. 2). By following the dashed line in Fig. 2 from negative to positive values of $a$, one can see that the trimer crosses the atom-dimer threshold at $a=a_{*}>0$, where one predicts an elastic atom-dimer resonance. At this point formation of deep dimer states (in this case called relaxation) in atom-dimer collisions is also enhanced for the same reason as above. According to [12, the atomdimer scattering resonance should be noticeable even in a purely atomic sample due to rescattering processes. Indeed, before leaving the trap, shallow dimers formed in the process of three-body recombination can collide with other atoms. The recombination rate itself is featureless around $a=a_{*}$, but the atom-dimer cross section in the vicinity of this point is highly $a$ dependent. Thus, at $a=a_{*}$ the three-body recombination can be enhanced in the sense that many more than three atoms are expelled from the trap leading to a measurable trap loss. We come back to this issue in Sec. V

The ratio of the two resonance positions, $a_{*} /\left|a_{-}\right|$, is of fundamental importance for studies of the universal three-body physics as in the universal limit it does not depend on the three-body parameter. In order to calculate this ratio we solve the bound-state Eq. (9) for $B_{t}=0$, $a<0$, and for $B_{t}=B_{d}, a>0$, with the same (arbitrary) cutoff $\Lambda$. The solid line in Fig. 3 shows $a_{*}^{(n)} /\left|a_{-}^{(n)}\right|$ as a function of the mass ratio $\delta$. Here we use the index $n$ introduced in Eq. (1) in order to emphasize that the values of $a_{*}$ and $a_{-}$are taken for one and the same Efimov state (connected by the dashed line in Fig. 2). The dashed line in Fig. 3 differs from the solid one by the scaling factor $\exp \left(\pi / s_{0}\right)$ and shows the ratio $a_{*}^{(n+1)} /\left|a_{-}^{(n)}\right|$. Note that 


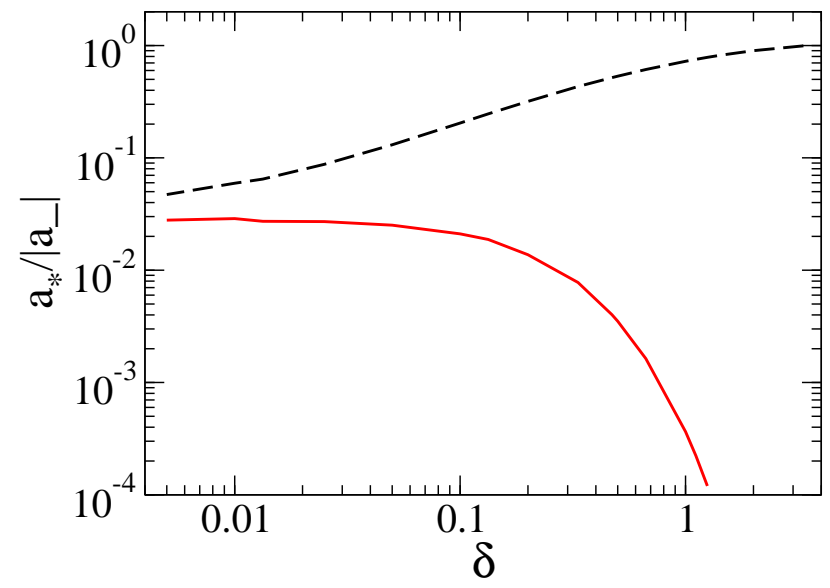

FIG. 3: (Color online) Solid line: $a_{*}^{(n)} /\left|a_{-}^{(n)}\right|$ vs $\delta$, where $n$ is the index of the Efimov state [see Eq. [1]]. Dashed line: $a_{*}^{(n+1)} /\left|a_{-}^{(n)}\right|=\exp \left(\pi / s_{0}\right) a_{*}^{(n)} /\left|a_{-}^{(n)}\right|$. (a)

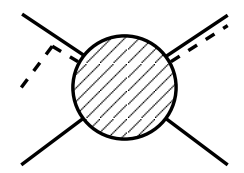

(b)

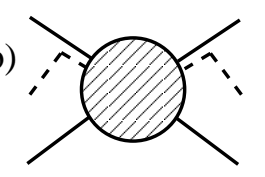

FIG. 4: Diagrammatic representation of (a) the three-body recombination amplitude and (b) the elastic three-body scattering. Line patterns are the same as in Fig. 1 .

the scaling factor rapidly increases with $\delta$ for $\delta \gtrsim 1$ and one can conclude that a sequence of Efimov resonances is more likely to be seen in systems with smaller mass ratios.

\section{B. Three-body recombination for $a>0$}

Let us now discuss the shapes of the inelastic loss resonances and calculate the three-body rate constants in a heteronuclear system. We first consider the case of positive scattering length, $a>0$, where the atoms can recombine into the shallow dimer and into deep dimers. The recombination into the shallow dimer can be related to the T-matrix element shown in Fig. 4(a). The event rate constant for inelastic scattering $\alpha$ is defined by the rate equation

$$
\frac{d}{d t} n_{2}=2 \frac{d}{d t} n_{1}=-2 \alpha n_{1} n_{2}^{2},
$$

where $n_{i}$ denotes the atomic number densities of the corresponding species.

The rate constant $\alpha_{s}$ for recombination into the shallow dimer is given by

$$
\alpha_{s}=4 \mu_{A D} \sqrt{\frac{\mu_{A D}}{\mu}} a^{2}\left|\mathcal{A}\left(0, k_{D} ; 0\right)\right|^{2}
$$

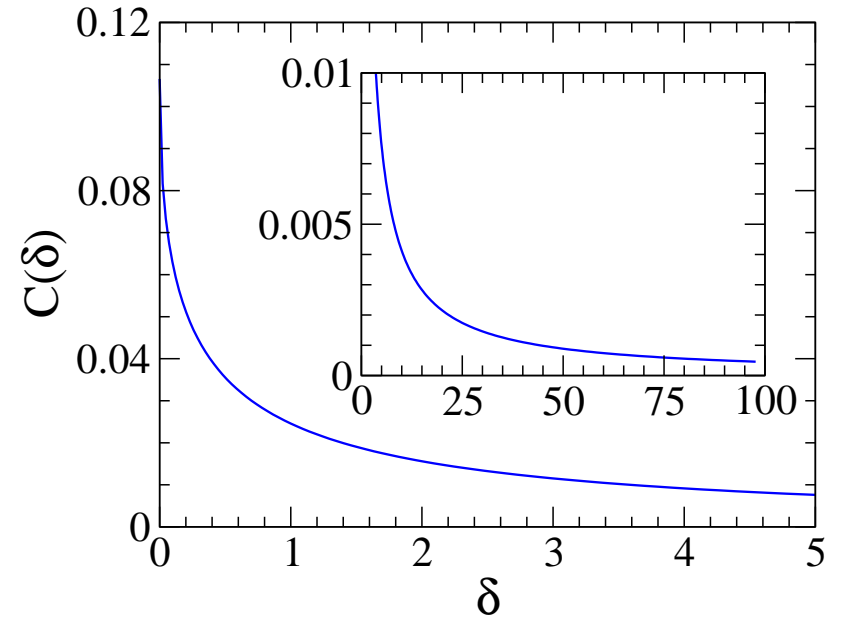

FIG. 5: (Color online) The coefficient $C$ for the different three-body recombination rates in dependence of the mass ratio $\delta=m_{1} / m_{2}$. The inset shows $C$ for larger values of $\delta$.

with the dimer breakup momentum $k_{D}=\sqrt{\mu_{A D} / \mu} a^{-1}$. If deep dimers are present, their effect on the recombination into the shallow dimer can be incorporated by analytically continuing the three-body parameter into the complex plane [18. We thus make the substitution

$$
\Lambda \rightarrow \Lambda \exp \left(i \eta_{*} / s_{0}\right)
$$

in Eq. (4), where $\eta_{*}$ accounts for the effect of the deep dimers. A nonzero value of $\eta_{*}$ also generates the width of the Efimov trimers. By evaluating Eq. (11) numerically, we find that the known analytical formula for the threeboson case 24 simply acquires a new mass-dependent overall coefficient. The modified analytical formula is hence

$$
\alpha_{s}=C(\delta) \frac{D\left(\sin ^{2}\left[s_{0} \ln \left(a / a_{0 *}\right)\right]+\sinh ^{2} \eta_{*}\right)}{\sinh ^{2}\left(\pi s_{0}+\eta_{*}\right)+\cos ^{2}\left[s_{0} \ln \left(a / a_{0 *}\right)\right]} \frac{\hbar a^{4}}{m_{1}},
$$

where $D=128 \pi^{2}(4 \pi-3 \sqrt{3})$ and the mass-dependent coefficient is denoted by $C(\delta)$. The parameter $a_{0 *}$ gives the position of the minimum in the three-body recombination. The coefficient $C(\delta)$ is shown in Fig. 5. The error in the extraction of $C(\delta)$ from fitting Eq. (13) to our numerical results for $\alpha_{s}$ is of order $10^{-3}$ for $\delta \leq 2$. For larger values of $\delta$ the numerical extraction of $C$ becomes difficult because of a very large value of the scaling factor. To depict $C(\delta)$ for $\delta \geq 2$ we use the analytical formula (56) derived in Sec. IV]

Although our calculations in this section are conducted by varying the complex three-body parameter $\Lambda$, we present the results in terms of the practically relevant length parameters $a_{-}<0, a_{*}>0$, and $a_{0 *}>0$, and the dimensionless elasticity parameter $\eta_{*}$. The universal theory predicts that the ratios $a_{*} /\left|a_{-}\right|$and $\left|a_{-}\right| / a_{0 *}$ depend only on the mass ratio $\delta$. The former is shown in Fig. 3 and the latter is $\left|a_{-}\right| / a_{0 *}=\exp \left(\pi / 2 s_{0}\right)$ as is derived in Sec. IV] This fixes the relative positions of all 
the three-body loss features on both sides of the Feshbach resonance.

The total rate of three-body recombination into all dimers (shallow and deep) for $a>0$ can be obtained from the optical theorem. It relates the imaginary part of the forward T-matrix element [shown in Fig. 4(b)] for vanishing momenta to the event rate constant of inelastic scattering, $\alpha$. This leads to the total recombination rate constant

$$
\alpha_{s}+\alpha_{d}=\operatorname{Im} T_{122 \rightarrow 122}=8 \pi a^{3} \operatorname{Im} \overline{\mathcal{A}}(0,0 ; 0),
$$

where $\overline{\mathcal{A}}$ denotes the appropriately infrared subtracted amplitude [24]:

$$
\begin{aligned}
& \overline{\mathcal{A}}(p, k ; E)=\mathcal{A}(p, k ; E)-\frac{4 \pi(1+\delta)}{m_{1} a p^{2}} \\
& \quad+\frac{4 \pi(1+\delta)^{2}}{m_{1} p} \arcsin [1 /(1+\delta)] \\
& \quad+\frac{8 a}{m_{1}}\left[(1+\delta)^{2} \arcsin [1 /(1+\delta)]-\sqrt{\delta(2+\delta)}\right] \ln p
\end{aligned}
$$

By subtracting Eq. 113 from Eq. 114 we find the rate constant for the recombination into deep dimers:

$$
\alpha_{d}=C(\delta) \frac{D \operatorname{coth}\left(\pi s_{0}\right) \cosh \left(\eta_{*}\right) \sinh \left(\eta_{*}\right)}{\sinh ^{2}\left(\pi s_{0}+\eta_{*}\right)+\cos ^{2}\left[s_{0} \ln \left(a / a_{0 *}\right)\right]} \frac{\hbar a^{4}}{m_{1}},
$$

where the coefficients $C(\delta)$ and $D$ are the same as in Eq. 13.

When $s_{0}$ is not too small such that $\exp \left(2 \pi s_{0}\right) \gg 1$ (see Table I), the denominators in Eqs. (13) and (16) are practically independent of $a$. In this case the $a$ dependence of $\alpha_{s}$ and $\alpha_{d}$ is simplified, and the corresponding expressions are known in the case of three identical bosons (see, for example, Ref. [1]).

\section{Atom-dimer scattering}

On the positive side of the Feshbach resonance $(a>$ 0 ) it is also possible to prepare an ultracold mixture of atoms and weakly bound dimers (see, for example, Refs. [10, 28]). An important observable in this case is the atom-dimer scattering length. Within our theory, it is given by

$$
a_{A D}=-\frac{\mu_{A D}}{2 \pi} \mathcal{A}\left(0,0 ;-\frac{1}{2 \mu a^{2}}\right),
$$

and its universal dependence on $a$ is parametrized by

$$
a_{A D}=\left(C_{1}(\delta)+C_{2}(\delta) \cot \left[s_{0} \ln \left(a / a_{*}\right)\right]\right) a,
$$

where the coefficients $C_{1}(\delta)$ and $C_{2}(\delta)$, calculated numerically, are shown in Fig. 6. Here we estimate the numerical error in the determination of $C_{1}(\delta)$ and $C_{2}(\delta)$ to be of order $10^{-3}$.

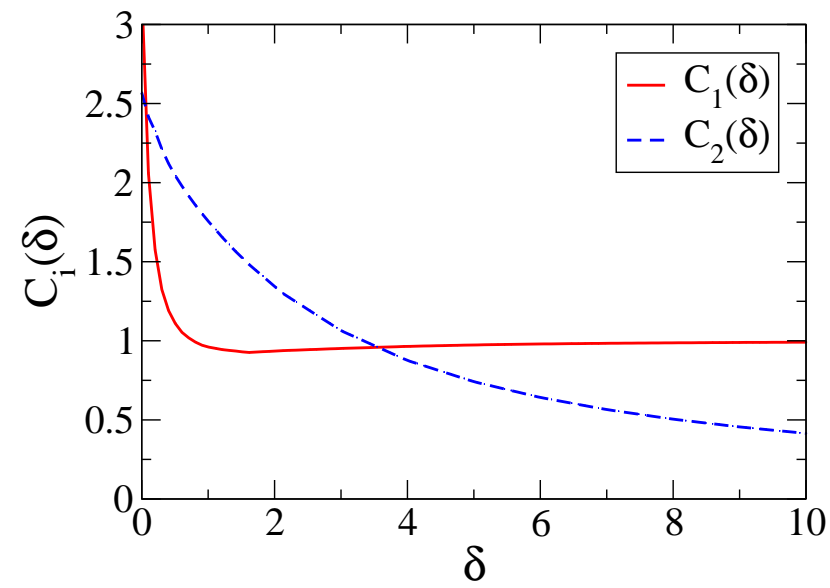

FIG. 6: (Color online) The parameters $C_{1}(\delta)$ and $C_{2}(\delta)$ in the expression for the atom-dimer scattering length, Eq. [18.

Efremov and collaborators have recently derived Eq. 18 for the atom-dimer scattering length in the Born-Oppenheimer approximation valid in the limit $\delta \rightarrow$ 0 31. For $\delta=0.081$, corresponding to the ${ }^{7} \mathrm{Li}^{-}{ }^{87} \mathrm{Rb}-{ }^{87} \mathrm{Rb}$ system, our values for the coefficients $C_{1}$ and $C_{2}$ agree with the ones given in Ref. 31] to within 2-3\% (see Table I). However, we observe a stronger discrepancy in between our value, $s_{0}=1.523$, and the Born-Oppenheimer result, $s_{0}=1.322$, for this system [31].

The effect of deep dimers on the atom-dimer scattering process can be incorporated by replacing $a_{*} \rightarrow$ $a_{*} \exp \left(-i \eta_{*} / s_{0}\right)$, equivalent to Eq. 12). At the scattering threshold, the atom-dimer relaxation rate constant $\beta$, defined by the rate equation

$$
\frac{d}{d t} n_{A}=\frac{d}{d t} n_{D}=-\beta n_{A} n_{D}
$$

is given by 8

$$
\begin{aligned}
& \beta\left(E=-B_{d}\right)=-\left(4 \pi \hbar / \mu_{A D}\right) \operatorname{Im} a_{A D} \\
& \quad=2 \pi C_{2}(\delta) \frac{\delta(\delta+2)}{\delta+1} \frac{\sinh \left(2 \eta_{*}\right)}{\sin ^{2}\left[s_{0} \ln \left(a / a_{*}\right)\right]+\sinh ^{2} \eta_{*}} \frac{\hbar a}{m_{1}} .
\end{aligned}
$$

Furthermore, we can calculate the atom-dimer relaxation rate constant above threshold. It is related to the inelastic atom-dimer scattering cross section by

$$
\beta(E)=\frac{k}{\mu_{A D}} \sigma_{A D}^{(\text {inel. })}(E),
$$

where $k=\sqrt{2 \mu_{A D}\left(E+B_{d}\right)}$. The energy dependent inelastic cross section is given by the difference of the total and the elastic cross sections,

$$
\sigma_{A D}^{(\text {inel. })}(E)=\frac{2 \mu_{A D}}{k} \operatorname{Im} \mathcal{A}(k, k ; E)-\frac{\mu_{A D}^{2}}{\pi}|\mathcal{A}(k, k ; E)|^{2} .
$$




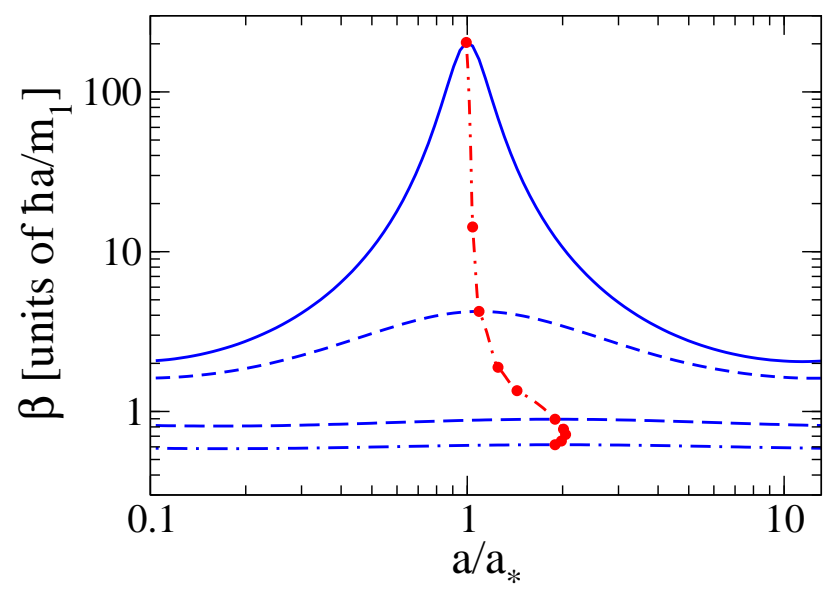

FIG. 7: (Color online) The dimer relaxation rate constant $\beta$ in units of $\hbar a / m_{1}$ for $\eta_{*}=0.1$ and $\delta=0.471$ as function of $a / a_{*}$. The solid, short-dashed, long-dashed, and dot-dashed lines show $\beta$ for $E / B_{d}=-1,-0.95,-0.5$, and 0 , respectively. The double-dot-dashed line indicates the trajectory of the resonance maximum as the energy is increased from $-B_{d}$ to zero.

We can use this formula up to the dimer breakup threshold at $k=k_{D}$ and thus map out the trajectory of the resonance peak. It moves from $a_{*}$ at the scattering threshold, $E=-B_{d}$, to $\left|a_{-}\right|$at the dimer breakup threshold, $E=0$. For $\delta<3.475$ the resonance peak moves to values $a>a_{*}$. Starting at $\delta=3.475$, where we have exactly $a_{*} /\left|a_{-}\right|=1$, it reverses this behavior and moves to values $a<a_{*}$. The peak height diminishes considerably with the energy. This effect is very large, especially for small values of $\delta$. For example, for $\eta_{*}=0.1$ and $\delta=0.1$, the peak at $E=0$ is smaller by a factor of 706 than the peak at $E=-B_{d}$. For $\delta=10$ this ratio still is 19.1. At $E=0$, we find excellent agreement with the analytical formula

$$
\begin{aligned}
& \beta(E=-0)=\pi[\delta(\delta+2)]^{3 / 2} /(\delta+1)^{2} \\
& \quad \times \frac{\sinh \left(2 \pi s_{0}\right) \sinh \left(2 \eta_{*}\right)}{\sinh ^{2}\left(\pi s_{0}+\eta_{*}\right)+\cos ^{2}\left[s_{0} \ln \left(a / a_{0 *}\right)\right]} \frac{\hbar a}{m_{1}},
\end{aligned}
$$

which is derived in Sec. IV] The peak position of $\beta(E=$ $-0)$ coincides exactly with the position of the maximum of the three-body recombination rate at threshold.

In Fig. 7) we show numerical results for $\beta$ for $\eta_{*}=0.1$ and $\delta=0.471$ corresponding to the K-Rb-Rb system observed in the Florence experiment [15. The solid, shortdashed, long-dashed, and dot-dashed lines show $\beta$ for $E / B_{d}=-1,-0.95,-0.5$, and 0 , respectively. As the energy is increased toward the breakup threshold, the resonance height decreases strongly and the resonance becomes less pronounced. The double-dot-dashed line shows the trajectory of the resonance maximum as the energy is increased from $-B_{d}$ to zero. As the energy is increased, the resonance position is shifted from $a_{*}$ toward larger values of $a$ until it reaches its maximum value of $2.04 a_{*}$ for $E / B_{d} \approx-0.25$. For larger energies, the res- onance position moves back to smaller values of $a$ and reaches $\left|a_{-}\right|=1.89 a_{*}$ at the dimer breakup threshold.

\section{Three-body recombination for $a<0$}

On the negative side of the Feshbach resonance, shallow dimers are absent and atoms can only recombine into deep dimers. The corresponding rate constant is again determined by using the optical theorem

$$
\alpha_{d}=\operatorname{Im} T_{122 \rightarrow 122}=8 \pi a^{3} \operatorname{Im} \overline{\mathcal{A}}(0,0 ; 0) .
$$

We have performed numerical calculations of $\alpha_{d}$ for mass ratios $\delta \leq 2$, where the numerical accuracy is better than $0.1 \%$. Our results agree with the formula

$$
\alpha_{d}=\frac{C(\delta)}{2} \frac{D \operatorname{coth}\left(\pi s_{0}\right) \sinh \left(2 \eta_{*}\right)}{\sin ^{2}\left[s_{0} \ln \left(a / a_{-}\right)\right]+\sinh ^{2}\left(\eta_{*}\right)} \frac{\hbar a^{4}}{m_{1}},
$$

where the coefficients $C(\delta)$ and $D$ are the same as in Eqs. 13 and (16). Equation 25) is derived in the next section.

\section{ANALYTICAL APPROACH}

Relying on an analysis of the recombination process in configuration space, Macek et al. [32, 33], Petrov [34, and Gogolin with co-workers [35] have obtained a completely analytic expression for the three-body recombination rate constant for identical bosons with a large positive scattering length $a$ when there are no deep dimers (i.e., $\eta_{*}=0$ ). Braaten and Hammer generalized this result to finite $\eta_{*}$ by making the analytic continuation $\kappa_{*} \rightarrow \kappa_{*} \exp \left(i \eta_{*} / s_{0}\right)$ in the amplitude of this process [1].

In this section we would like to present an approach that allows one to calculate almost all zero-energy threebody observables analytically [in particular, $\alpha_{s}, \alpha_{d}(a>$ $0)$, and $\alpha_{d}(a<0)$ ] for heteronuclear Bose-Fermi or BoseBose mixtures. We generalize the method introduced in Ref. [36] for fermionic mixtures and present it in more detail, also because it is conceptually different from the approaches of Refs. [32, 33, 35].

Let us consider the process of three-body recombination into the shallow heteronuclear dimer. When the total energy of the system, $E$, becomes smaller than the binding energy of the dimer, the three-body recombination rate tends to a finite zero-energy value. In order to calculate this quantity we work in the coordinate representation. It is instructive to derive the real-space ana$\log$ of Eq. (4) directly from the Schrödinger equation. At $E=0$ the latter reads

$$
\left[\sum_{i=1,2}\left(-\frac{\hbar^{2} \nabla_{\mathbf{R}_{\mathbf{i}}}^{2}}{2 m_{2}}+U\left(\left|\mathbf{r}-\mathbf{R}_{\mathbf{i}}\right|\right)\right)-\frac{\hbar^{2} \nabla_{\mathbf{r}}^{2}}{2 m_{1}}\right] \Psi=0,
$$


where $\mathbf{R}_{\mathbf{i}}$ are the coordinates of the bosons and $\mathbf{r}$ is the coordinate of the third atom. For the interspecies interaction we use the zero-range Fermi pseudopotential

$$
U\left(\left|\mathbf{r}-\mathbf{R}_{\mathbf{i}}\right|\right)=\frac{2 \pi \hbar^{2} a}{\mu} \delta\left(\mathbf{r}-\mathbf{R}_{\mathbf{i}}\right) \frac{\partial}{\partial\left|\mathbf{r}-\mathbf{R}_{\mathbf{i}}\right|}\left(\left|\mathbf{r}-\mathbf{R}_{\mathbf{i}}\right| \cdot\right) .
$$

Let us temporarily adopt the units $\hbar=2 \mu=$ $a=1$, separate the center-of-mass motion, and introduce the new coordinates $\mathbf{x}=\left[\mathbf{R}_{\mathbf{1}} \sin \phi-\mathbf{R}_{\mathbf{2}}+\right.$ $\mathbf{r}(1-\sin \phi)] / \cos \phi$ and $\mathbf{y}=\mathbf{r}-\mathbf{R}_{\mathbf{1}}$, where $\phi$ is defined in Eq. 88. The bosonic symmetry condition, $\Psi\left(\mathbf{R}_{1}, \mathbf{R}_{2}, \mathbf{r}\right)=\Psi\left(\mathbf{R}_{2}, \mathbf{R}_{1}, \mathbf{r}\right)$, in the new coordinates reads

$$
\Psi(\mathbf{x}, \mathbf{y})=\Psi(-\mathbf{x} \sin \phi+\mathbf{y} \cos \phi, \mathbf{x} \cos \phi+\mathbf{y} \sin \phi),
$$

and Eq. (26) takes the form

$$
\begin{aligned}
\left(-\nabla_{\mathbf{x}}^{2}-\nabla_{\mathbf{y}}^{2}\right) \Psi(\mathbf{x}, \mathbf{y})=f(\mathbf{x}) \delta(\mathbf{y}) & \\
+ & f(-\mathbf{x} \sin \phi+\mathbf{y} \cos \phi) \delta(\mathbf{x} \cos \phi+\mathbf{y} \sin \phi),
\end{aligned}
$$

where we define

$$
f(\mathbf{x}):=-4 \pi \lim _{y \rightarrow 0} \partial[y \Psi(\mathbf{x}, \mathbf{y})] / \partial y .
$$

From Eqs. 290 and (30) it is easy to see that

$$
\Psi(\mathbf{x}, \mathbf{y}) \underset{y \rightarrow 0}{\longrightarrow} f(\mathbf{x})(1 / y-1) / 4 \pi .
$$

Thus, the function $f$ describes the motion of an atom relative to the other two atoms when they are on top of each other. In some cases it is useful to consider $f$ as an atom-dimer wave function. Indeed, for large $x$ expressions $\Psi \propto \phi_{b}(y) \exp (i x) / x$ and $f(x) \propto(8 \pi)^{1 / 2} \exp (i x) / x$ equivalently describe the relative outgoing motion of the products of three-body recombination. The coefficient $(8 \pi)^{1 / 2}$ is obtained by comparing Eq. (31) and the small$y$ asymptote of the normalized molecular wave function

$$
\phi_{b}(y)=\exp (-y) / \sqrt{2 \pi} y \underset{y \rightarrow 0}{\longrightarrow}(1 / y-1) / \sqrt{2 \pi} .
$$

Therefore, in order to calculate the three-body recombination rate constant we first derive and solve an equation for $f$, then separate the large- $x$ asymptote $f \propto$ $\exp (i x) / x$, and finally relate the coefficient in front of it to the three-body recombination rate constant.

Using the Green's function $G(X)=1 / 4 \pi^{3} X^{4}$ of Eq. 29) we can express $\Psi$ through $f$ :

$$
\begin{aligned}
& \Psi(\mathbf{x}, \mathbf{y})=\Psi_{0}+\int\left[G\left(\sqrt{\left(\mathbf{x}-\mathbf{x}^{\prime}\right)^{2}+y^{2}}\right)\right. \\
& \left.\quad+G\left(\sqrt{\left(\mathbf{x}+\mathbf{x}^{\prime} \sin \phi\right)^{2}+\left(\mathbf{y}-\mathbf{x}^{\prime} \cos \phi\right)^{2}}\right)\right] f\left(\mathbf{x}^{\prime}\right) d^{3} x^{\prime},
\end{aligned}
$$

where $\Psi_{0}$ is a solution of the homogeneous Euler equation $\left(-\nabla_{\mathbf{x}}^{2}-\nabla_{\mathbf{y}}^{2}\right) \Psi_{0}=0$ without singularities. In the case of three-body recombination, $\Psi_{0}$ is a correctly normalized wave function of three free atoms. We consider the atoms in a volume $V$ in a state where the two bosons are not in the same quantum state (cold thermal gas). Then, in the region relevant for the recombination $(x \lesssim 1, y \lesssim 1)$, we get

$$
\Psi_{0}=\sqrt{2} V^{-3 / 2} .
$$

We point out that $\Psi_{0}$ should be set to zero for the problem of atom-dimer scattering just below the breakup threshold $(E=-0)$ as in this case the atoms cannot move freely at large distances.

The equation for $f$ is obtained by substituting Eq. (33) into Eq. (30). We write it in the form

$$
(\hat{L}-1) f(\mathbf{x})=4 \pi \Psi_{0},
$$

where the integral operator $\hat{L}$ is defined as

$$
\begin{array}{r}
\hat{L} f(\mathbf{x})=4 \pi \int\left\{G\left(\left|\mathbf{x}-\mathbf{x}^{\prime}\right|\right)\left[f(\mathbf{x})-f\left(\mathbf{x}^{\prime}\right)\right]\right. \\
\left.-G\left(\sqrt{x^{2}+x^{\prime 2}+2 \mathbf{x x}^{\prime} \sin \phi}\right) f\left(\mathbf{x}^{\prime}\right)\right\} d^{3} x^{\prime} .
\end{array}
$$

The operator $\hat{L}$ conserves angular momentum, and Eq. (35) can be written as a set of uncoupled equations for each spherical harmonic of $f(\mathbf{x})$. For the three-body recombination problem at hand we look for a spherically symmetric solution $f(\mathbf{x})=f(x)$. Then, Eq. (36) reduces to

$$
\begin{aligned}
\hat{L} f(x) & =\frac{4}{\pi} \int_{0}^{\infty}\left[\frac{f\left(x^{\prime}\right)-f(x)}{\left(x^{2}-x^{\prime 2}\right)^{2}}\right. \\
& \left.+\frac{f\left(x^{\prime}\right)}{\left(x^{2}+x^{\prime 2}\right)^{2}-4 x^{2} x^{\prime 2} \sin ^{2} \phi}\right] x^{\prime 2} d x^{\prime} .
\end{aligned}
$$

The integrals in Eqs. (36) and (37) are taken as principal values [37.

Let us now discuss the structure of possible solutions of Eq. (35). Obviously, $f(x)$ is a sum of a particular solution of the inhomogeneous Eq. (35) and a general solution of the homogeneous equation

$$
(\hat{L}-1) \chi(x)=0 .
$$

Physically, Eq. (38) describes the atom-dimer channel just below the dimer breakup threshold $\left(\Psi_{0}=0\right)$. Therefore, at distances $x \gg 1$, the function $\chi(x)$ is a linear combination of $\exp (i x) / x$ and $\exp (-i x) / x$.

In order to understand the short-distance behavior of $\chi(x)$, note the following property of the operator $\hat{L}$ :

$$
\hat{L} x^{\nu}=\lambda(\nu) x^{\nu-1}
$$

for any complex exponent $\nu$ in the region $-3<\operatorname{Re}(\nu)<$ 1 , which is, in fact, the region of convergence of the integral on the left-hand side of Eq. 393 [38. The function $\lambda(\nu)$ is given by

$$
\lambda(\nu)=-(\nu+1) \tan \frac{\pi \nu}{2}-\frac{2 \sin [\phi(\nu+1)]}{\sin (2 \phi) \cos (\pi \nu / 2)}
$$


and has two complex conjugate roots, $\nu_{1,2}=-1 \pm i s_{0}$, where $s_{0}$ is a real number satisfying Eq. (7). At short distances the operator $\hat{L}$ in Eq. 38 dominates over 1, and any solution of this equation should be a linear superposition of $\chi \propto x^{-1+i s_{0}}$ and its complex conjugate. From now on we use the notation $\chi$ for the solution of Eq. 38 with the following asymptotes:

$$
\chi(x)=\left\{\begin{array}{lr}
A x^{\nu}=A x^{-1+i s_{0}}, & x \ll 1 \\
x^{-1} e^{i x+i \sigma-h}+x^{-1} e^{-i x-i \sigma+h}, & x \gg 1,
\end{array}\right.
$$

where $A$ is a complex number, and $\sigma$ and $h$ are real numbers. The physical solution of Eq. (38) (i.e., the one corresponding to a given three-body parameter) is expressed as

$$
\chi_{\theta}(x)=e^{i \theta} \chi(x)+e^{-i \theta} \chi^{*}(x),
$$

where $\theta$ is the three-body parameter (a complex number with imaginary part $\eta_{*}$ ).

The normalization in Eq. (41) is chosen such that

$$
\left\langle p \chi(p x) \mid p^{\prime} \chi\left(p^{\prime} x\right)\right\rangle=\int_{0}^{\infty} p \chi(p x) p^{\prime} \chi\left(p^{\prime} x\right) x^{2} d x=2 \pi \delta\left(p-p^{\prime}\right) .
$$

The first equality in Eq. (43) is our definition of the scalar product (note the absence of the complex conjugation), and the second equality follows from the fact that $p \chi(p x)$ and $p^{\prime} \chi\left(p^{\prime} x\right)$ are eigenfunctions of the symmetric operator $\hat{L}$ corresponding to the eigenvalues $p$ and $p^{\prime}$. They are orthogonal for $p \neq p^{\prime}$ and their scalar product in the vicinity of $p=p^{\prime}$ can be worked out in the same way as in Ref. 39] (see Sec. 21 there). A simple change of the integration variable in Eq. (43) leads to the completeness condition

$$
\int_{0}^{\infty} p^{2} \chi(p x) \chi\left(p x^{\prime}\right) d p=2 \pi \delta\left(x-x^{\prime}\right) / x^{2} .
$$

Equations (43) and (44) allow us to construct the integral operator $(\hat{L}-1)^{-1}$ needed to solve Eq. 35$)$. In order to avoid problems with divergence of the corresponding integrals let us introduce an auxiliary function $g_{0}(x)$ related to $f(x)$ by

$$
f(x)=4 \pi \Psi_{0}\left[-1-\lambda(0) / x+\lambda(0) \lambda(-1) g_{0}(x)\right] .
$$

Substituting this expression into Eq. (35) and using Eq. 39$)$, we find that $g_{0}(x)$ satisfies the equation $(\hat{L}-$ 1) $g_{0}(x)=x^{-2}$. Applying the operator $(\hat{L}-1)^{-1}$ to $x^{-2}$ we obtain the following particular solution:

$$
\begin{aligned}
g_{0}(x) & =\frac{1}{2 \pi x} \int_{0}^{\infty} \chi(z) d z \\
& \times\left[\int_{0}^{\infty} \frac{\chi(y) y d y}{y-x-i 0}-\frac{2 \pi i x \chi(x)}{1-\exp \left(-2 \pi s_{0}\right)}\right],
\end{aligned}
$$

where the first integral is defined as

$$
\int_{0}^{\infty} \chi(z) d z=\lim _{\epsilon \rightarrow+0} \int_{0}^{\infty} \chi(z) z^{\epsilon} d z .
$$

The rule of going around the pole in the second integral and the numerical coefficient in front of the second term in the square brackets on the right-hand side of Eq. 46 regulate the entry of $\chi(x)$, which can be arbitrary, into the particular solution $g_{0}(x)$. Using this freedom, we choose these parameters in such a way that $g_{0}(x)$ does not contain oscillating terms proportional to $x^{-1+i s_{0}}$ at small $x$. Direct calculation shows that in the limit $x \rightarrow 0$ the right-hand side of Eq. (46) equals $g_{0}(x) \approx\left[\int_{0}^{\infty} \chi(z) d z\right]^{2} / 2 \pi x$ to the leading order in $x$. On the other hand, according to Eq. (39), the same quantity in the same limit can be written as $g_{0}(x)=$ $(\hat{L}-1)^{-1} x^{-2} \approx \hat{L}^{-1} x^{-2}=1 /[\lambda(-1) x]$, which leads to the result

$$
\int_{0}^{\infty} \chi(z) d z=\sqrt{2 \pi / \lambda(-1)} .
$$

Another consequence of our choice of the particular solution (46) is that removing the oscillating terms from $g_{0}(x)$ makes it real, since any imaginary part of $g_{0}$ would necessarily be a solution of the homogeneous Eq. (38). Therefore, $g_{0}$ would have oscillations at short $x$, the absence of which we have ensured. Clearly, the function $f$ obtained by virtue of Eq. (45) is also real. Moreover, property $(39)$ ensures that $f=o(1)$ at small $x$ (i.e., its Taylor expansion starts with $x^{1}$ at least). Therefore, this solution of Eq. (35) is not sensitive to the short-range physics and does not depend on the three-body parameter.

Integrating Eq. (46) in the limit $x \gg 1$ we get

$$
g_{0}(x) \underset{x \rightarrow \infty}{\longrightarrow} \frac{-i}{\sinh \left(\pi s_{0}\right)} \sqrt{\frac{2 \pi}{\lambda(-1)}} \frac{\cos \left[x+\sigma+i\left(h+\pi s_{0}\right)\right]}{x} .
$$

It can be real only if $h=-\pi s_{0}$ [note that $\left.\lambda(-1)<0\right]$; compare Ref. 32.

Finally, the result that we are interested in is the linear combination

$$
f_{\theta}(x)=f(x)+\gamma \chi_{\theta}(x),
$$

where the complex number $\gamma$ is chosen such that $f_{\theta}(x)$ contains only an outgoing wave at large $x$ (corresponding to an atom and a dimer flying apart after the three-body recombination event). This condition gives

$$
\gamma=i \frac{\pi \Psi_{0} \lambda(0) \sqrt{2 \pi \lambda(-1)}}{\sinh \left(\pi s_{0}\right) \cosh \left(\pi s_{0}-i \theta\right)} .
$$

Keeping only the relevant oscillating term at large $x$, we obtain

$$
f_{\theta}(x) \underset{x \rightarrow \infty}{\longrightarrow} i 4 \gamma \sin \theta \sinh \left(\pi s_{0}\right) \exp (i x+i \sigma) / x .
$$

Equation (52) together with Eqs. (31) and $(32)$ gives us the atom-dimer outgoing flux. Indeed, the large- $x$ asymptote $f=\xi \exp (i x) / x$, where $\xi$ is any complex amplitude, is accompanied by the flux $|\xi|^{2} \Phi_{\infty}$, where

$$
\Phi_{\infty}=2 \times(8 \pi)^{-1} \times(4 \pi) \times 2=2 .
$$


Here we have explicitly written out the following factors: the factor of 2 reflects the two symmetric possibilities of forming the dimer (corresponding to the interchange $\left.\mathbf{R}_{\mathbf{1}} \rightleftarrows \mathbf{R}_{\mathbf{2}}\right)$, the factor of $(8 \pi)^{-1}$ arises from the relation in between $\Psi$ and $f$ [see the discussion preceding Eq. [32 ], the factor of $4 \pi$ is the solid angle in the outgoing atom-dimer channel, and the last factor of 2 is the atom-dimer relative velocity in the $x, y$ coordinates. The three-body recombination rate constant $\alpha_{s}$ is obtained by taking the squared modulus of the prefactor in front of $\exp (i x+i \sigma) / x$ in Eq. (52) and by multiplying it by $\Phi_{\infty}$, by the factor of $1 / 2$, reflecting the fact that the number of pairs in the gas of species 2 is $n_{2}^{2} / 2$, and by the factor $\hbar a^{4} / 2 \mu$ in order to restore the original physical units. We should also mention that the nine-dimensional volume $V^{3}$ is taken to be a unit volume in the original system of coordinates $\left\{\mathbf{r}, \mathbf{R}_{\mathbf{1}}, \mathbf{R}_{\mathbf{2}}\right\}$. In the new coordinates $\left\{\mathbf{x}, \mathbf{y}, \mathbf{R}_{\text {c.m. }}\right\}$, where $\mathbf{R}_{\text {c.m. }}$ is the center-of-mass coordinate, this volume is $V^{3}=\cos ^{-3} \phi$. The final result for the three-body recombination rate constant reads

$$
\begin{aligned}
\alpha_{s} & =\frac{32 \pi^{3} \cos ^{3} \phi \lambda^{2}(0)|\lambda(-1)|}{\sin \phi} \\
& \times \frac{\sin ^{2}\left[s_{0} \ln \left(a / a_{* 0}\right)\right]+\sinh ^{2} \eta_{*}}{\sinh ^{2}\left(\pi s_{0}+\eta_{*}\right)+\cos ^{2}\left[s_{0} \ln \left(a / a_{0 *}\right)\right]} \frac{\hbar a^{4}}{m_{1}},
\end{aligned}
$$

where we have expressed the three-body parameter $\theta$ through the original physical units:

$$
\theta=s_{0} \ln \left(a / a_{* 0}\right)+i \eta_{*} .
$$

Formula (54) is in excellent agreement with our numerical results. Comparison with Eq. (13) leads to [40]

$$
C(\delta)=\frac{(1+\delta)^{2} \arcsin [1 /(1+\delta)]-\sqrt{\delta(2+\delta)}}{2(4 \pi-3 \sqrt{3})} .
$$

As explained in Sec. III the constant of the three-body recombination into deep dimers can be derived from the optical theorem. The result for $\alpha_{d}$ is given by Eq. 116. Here we would like to show how one can derive this result by using the method of this section. In contrast to the recombination into shallow states we now have to look at the balance of the incoming and outgoing fluxes of atoms corresponding to the short-distance asymptote of $f_{\theta}(x)$ given by Eq. (50)

$$
f_{\theta}(x) \underset{x \rightarrow 0}{\longrightarrow} \gamma \chi_{\theta}(x)=\gamma\left(A e^{i \theta} x^{-1+i s_{0}}+A^{*} e^{-i \theta} x^{-1-i s_{0}}\right),
$$

In analogy with $\Phi_{\infty}$, let $\Phi_{0}$ denote the number of atom triples disappearing at the origin $(\mathbf{x}=0, \mathbf{y}=0)$, provided the function $f$ takes the form of the incoming wave $x^{-1-i s_{0}}$ with unit weight. With this definition, the recombination rate constant follows from Eq. (57):

$$
\alpha_{d}=\left(\hbar a^{4} / 2 \mu\right)|\gamma|^{2} \Phi_{0}|A|^{2} \sinh \left(2 \eta_{*}\right) .
$$

The product $\Phi_{0}|A|^{2}$ can easily be found from definition (41) by equating the fluxes at $x \rightarrow 0$ and at $x \rightarrow \infty$ and using Eq. (53):

$$
\Phi_{0}|A|^{2}=2 \Phi_{\infty} \sinh \left(2 \pi s_{0}\right)=4 \sinh \left(2 \pi s_{0}\right) .
$$

Substituting Eqs. (59) and (51) into Eq. (58), we obtain Eq. (16) exactly. We point out that it is in principle possible to obtain $\Phi_{0}$, and, therefore, $|A|^{2}$, by substituting the expression $f=x^{-1-i s_{0}}$ into Eq. (33) and calculating the incoming flux from the resulting wave function $\Psi$ in the six-dimensional configurational $\{\mathbf{x}, \mathbf{y}\}$-space.

Our analytical approach can also be used to derive $\alpha_{d}$ on the negative side of the resonance $(a<0)$. In this case we use the units $\hbar=|a|=2 \mu=1$, and some equations described above should be modified accordingly. In particular, Eq. (35) reads

$$
(\hat{L}+1) \tilde{f}(x)=4 \pi \Psi_{0}
$$

and we now introduce an auxiliary function $\tilde{g}_{0}$ related to $\tilde{f}$ by

$$
\tilde{f}(x)=4 \pi \Psi_{0}\left[1-\lambda(0) / x+\lambda(0) \lambda(-1) \tilde{g}_{0}(x)\right],
$$

where $\tilde{g}_{0}$ satisfies $(\hat{L}+1) \tilde{g}_{0}(x)=x^{-2}$. We write the solution in the form

$$
\tilde{g}_{0}(x)=\frac{1}{2 \pi x} \int_{0}^{\infty} \chi(z) d z \int_{0}^{\infty} \frac{\chi(y) y d y}{y+x}
$$

and integrating it in the small- $x$ limit we get the asymptote

$$
\tilde{f}(x) \underset{x \rightarrow 0}{\longrightarrow} i \frac{2 \pi \Psi_{0} \lambda(0) \sqrt{2 \pi \lambda(-1)}}{\sinh \left(\pi s_{0}\right)} A x^{-1+i s_{0}} .
$$

The function $\tilde{f}$ is a solution of Eq. 60, but its oscillations at small $x$ do not have (in general) the correct phase imposed by Eq. (41). This difficulty is resolved by observing that $\tilde{f}^{*}$ also satisfies Eq. 600. The correctly behaving solution reads

$$
\tilde{f}_{\theta}(x)=\frac{\exp (i \theta) \tilde{f}(x)+\exp (-i \theta) \tilde{f}^{*}(x)}{\exp (i \theta)+\exp (-i \theta)},
$$

and by subtracting the outgoing flux from the incoming one at small $x$ we obtain the result, cf. Eq. 25,

$$
\begin{aligned}
\alpha_{d}(a<0) & =16 \pi^{3} \cos ^{3} \phi \lambda^{2}(0)|\lambda(-1)| \operatorname{coth}\left(\pi s_{0}\right) / \sin \phi \\
& \times \frac{\sinh \left(2 \eta_{*}\right)}{\cos ^{2}\left[s_{0} \ln \left(|a| / a_{0 *}\right)\right]+\sinh ^{2} \eta_{*}} \frac{\hbar a^{4}}{m_{1}}
\end{aligned}
$$

Equation 65) also gives the ratio

$$
\left|a_{-}\right| / a_{0 *}=\exp \left(\pi / 2 s_{0}\right) .
$$

In other words, the maxima of $\alpha_{s}$ and $\alpha_{d}(a>0)$ and the maxima of $\alpha_{d}(a<0)$ are symmetric with respect to the center of the Feshbach resonance.

Let us now return to the case $a>0$ and discuss some properties of atom-dimer collisions just below the dimer breakup threshold $(E=-0)$. Namely, by substituting the large- $x$ asymptote of $\chi$ into Eq. 42 one readily obtains the $s$-wave contribution to the scattering $S$-matrix

$$
S_{0}=-e^{2 i \sigma} \cosh \left(\pi s_{0}+i \theta\right) / \cosh \left(\pi s_{0}-i \theta\right) .
$$


From this expression, one can calculate the atom-dimer inelastic rate constant at zero total energy:

$$
\begin{aligned}
& \beta(E=-0)=\pi \cos ^{2} \phi \cot \phi \\
& \quad \times \frac{\sinh \left(2 \pi s_{0}\right) \sinh \left(2 \eta_{*}\right)}{\sinh ^{2}\left(\pi s_{0}+\eta_{*}\right)+\cos ^{2}\left[s_{0} \ln \left(a / a_{0 *}\right)\right]} \frac{\hbar a}{m_{1}},
\end{aligned}
$$

see also Eq. (23). Remarkably, $\beta(E=-0)$ also reaches its maximum at $a=\left|a_{-}\right|$, and for small $s_{0}$ the peak can be quite narrow. We conjecture that this behavior is due to the Efimov state that crosses the atom-dimer threshold at $a=a_{*}$ and then exists as a scattering resonance. Our numerical results support this conjecture. We find that for mass ratios $\delta<3.475$ the resonance peak moves to values $a>a_{*}$, while it moves to values $a<a_{*}$ for $\delta>3.475$. The resonance peak then hits the three-atom threshold at $a=\left|a_{-}\right| \exp \left(-\pi / s_{0}\right)$. In Fig. 7, we have shown $\beta$ for the specific case $\eta_{*}=0.1$ and $\delta=0.471$ corresponding to the K-Rb-Rb system observed in the Florence experiment [15. The trajectory of the scattering resonance is given by the double-dot-dashed line in the figure. For smaller $s_{0}$ leading to a larger scaling factor, we conclude that the center of this Efimov scattering resonance can travel quite far from the value $a=a_{*}$ at $E=-B_{d}$ to the value $a=\left|a_{-}\right| \exp \left(-\pi / s_{0}\right)$ at $E=0$. We have verified this behavior numerically. It is thus worth investigating this scattering resonance in the vicinity of the atom-dimer threshold experimentally.

\section{COMPARISON TO EXPERIMENT}

There are several experiments on heteronuclear BoseBose and Bose-Fermi mixtures, to which our results are directly applicable (in the universal limit). In Table I, we present the universal predictions for some combinations of alkali isotopes being investigated at the moment and interesting from the viewpoint of Efimov few-body physics. We sort them by the value of the scaling factor.

\section{A. The ${ }^{40} \mathrm{~K}-{ }^{87} \mathbf{R b}$ mixture}

Zirbel et al. at JILA recently studied weakly bound fermionic ${ }^{40} \mathrm{~K}-{ }^{87} \mathrm{Rb}$ molecules and their stability in collisions with atoms near a wide (open-channel-dominated) heteronuclear Feshbach resonance at $B_{0}=546.7 \mathrm{G}$ [28]. In particular, they measured the atom-dimer relaxation rate for collisions of these dimers with $\mathrm{Rb}$ atoms as a function of $a$. The corresponding data (see Fig. 2 in Ref. 28] ) can be fit very well with our Eq. 20, where the fitting parameters are $a_{*}=200 \pm 50 a_{B o h r}$ and $\eta_{*}=0.05 \pm 0.02$. In the same work, the authors also measured the three-body recombination rate constant on both sides of this Feshbach resonance (i.e., in the same universal region). We fit their results with Eq. 25 on the negative side of the resonance and with the sum $\alpha_{s}+\alpha_{d}$ given by Eqs. 13 and 16 on the positive side 41]. A good agreement is achieved if we choose $a_{*}=300 \pm 100 a_{B o h r}$ (for this mass ratio $a_{-}=-1.96 a_{*}$, see Table If and the same $\eta_{*}$ as above. These parameter values lead to a peak of the three-body recombination at $a=a_{-} \approx-600 a_{\text {Bohr }}$. Although in Ref. 28. the peak has not been identified, the overall shapes of $\beta$ and $\alpha$ measured for this particular Feshbach resonance indicate that it is worth performing a more detailed measurement of the three-body loss rate around this value of $a$.

\section{B. The ${ }^{87} \mathbf{R b}-{ }^{41} \mathbf{K}$ mixture}

The group of Inguscio and Minardi in Florence investigated a Bose-Bose mixture of ${ }^{87} \mathrm{Rb}$ and ${ }^{41} \mathrm{~K}[15]$. They observed three loss resonances by scanning the scattering length and monitoring the population dynamics of the species in the vicinity of each of the resonances. For negative scattering length, they identified a $\mathrm{K}-\mathrm{Rb}-\mathrm{Rb}$ resonance at $a=-246 a_{B o h r}$ and a K-K-Rb resonance at $a=-22000 a_{B o h r}$. The third resonance is observed at the positive scattering length $a=667 a_{B o h r}$ and attributed to enhanced atom-dimer scattering in the K$\mathrm{Rb}-\mathrm{Rb}$ three-body system. This process is assumed to contribute to three-body losses through multiple rescattering processes (see also Ref. [12]). An independent confirmation of this resonance in a system prepared directly out of $\mathrm{K}-\mathrm{Rb}$ dimers and $\mathrm{Rb}$ atoms would be desirable. Unfortunately, in contrast to the JILA experiment, the dimers are bosonic and their short lifetime can make such a confirmation difficult 42 .

The interspecies van der Waals length in the K-Rb system is $\ell_{v d W}=72 a_{B o h r}$, such that these resonances should be within the range of validity of the universal theory. Assuming that the observed K-Rb-Rb features are due to Efimov resonances, one can extract the ratio $a_{*} /\left|a_{-}\right|=2.7$ from the Florence experiment, whereas our theory predicts $a_{*} /\left|a_{-}\right|=0.52$. The discrepancy can be attributed to the effective range corrections. In particular, one should be careful with the feature at $a=-246 a_{B o h r}$, which is not too large compared to the van der Waals length. Besides, if we believe in the "rescattering" nature of the positive- $a$ resonance, one should take into account a finite-energy shift of the position of the atom-dimer scattering resonance. Indeed, even at zero temperature, dimers formed by three-body recombination collide with stationary $\mathrm{Rb}$ atoms at the finite collision energy $\left[m_{R b}^{2} /\left(m_{K}+2 m_{R b}\right)^{2}\right] B_{d} \approx 0.16 B_{d}$. In Fig. 7, we have shown numerical results for $\beta$ for energies from the scattering threshold, $E=-B_{d}$, up to the breakup threshold, $E=0$, using $\eta_{*}=0.1$ and $\delta=0.471$ corresponding to the $\mathrm{K}-\mathrm{Rb}-\mathrm{Rb}$ resonance at $a=667 a_{B o h r}$. At $E=0$, the resonance peak is only $7 \%$ higher than the minimum value of $\beta$ and the resonance is almost completely washed out. Moreover, the peak value of $\beta$ at $E=0$ is a factor of 300 smaller than at $E=-B_{d}$. 


\begin{tabular}{|l||l|l|l|l|l|l|}
\hline & ${ }^{7}$ Li-Cs-Cs & ${ }^{6}$ Li-Rb-Rb & ${ }^{7}$ Li-Rb-Rb & ${ }^{40} \mathrm{~K}-\mathrm{Rb}-\mathrm{Rb}$ & ${ }^{41} \mathrm{~K}-\mathrm{Rb}-\mathrm{Rb}$ & $\mathrm{Rb}^{41} \mathrm{~K}^{41} \mathrm{~K}$ \\
\hline \hline$\delta$ & 0.053 & 0.069 & 0.081 & 0.460 & 0.471 & 2.21 \\
$s_{0}$ & 1.850 & 1.635 & 1.523 & 0.6536 & 0.6444 & 0.2462 \\
$\exp \left(\pi / s_{0}\right)$ & 5.465 & 6.835 & 7.864 & 122.7 & 131.0 & 348000 \\
$a_{*} /\left|a_{-}\right|$ & 0.13 & 0.16 & 0.18 & 0.51 & 0.52 & 0.91 \\
$C(\delta)$ & 0.072 & 0.068 & 0.066 & 0.037 & 0.037 & 0.015 \\
$C_{1}(\delta)$ & 2.54 & 2.33 & 2.22 & 1.14 & 1.13 & 0.94 \\
$C_{2}(\delta)$ & 2.52 & 2.5 & 2.47 & 2.08 & 2.07 & 1.30 \\
\hline
\end{tabular}

TABLE I: Universal parameters for various heteronuclear mixtures. The isotopes of rubidium and cesium are ${ }^{87} \mathrm{Rb}$ and ${ }^{133} \mathrm{Cs}$.

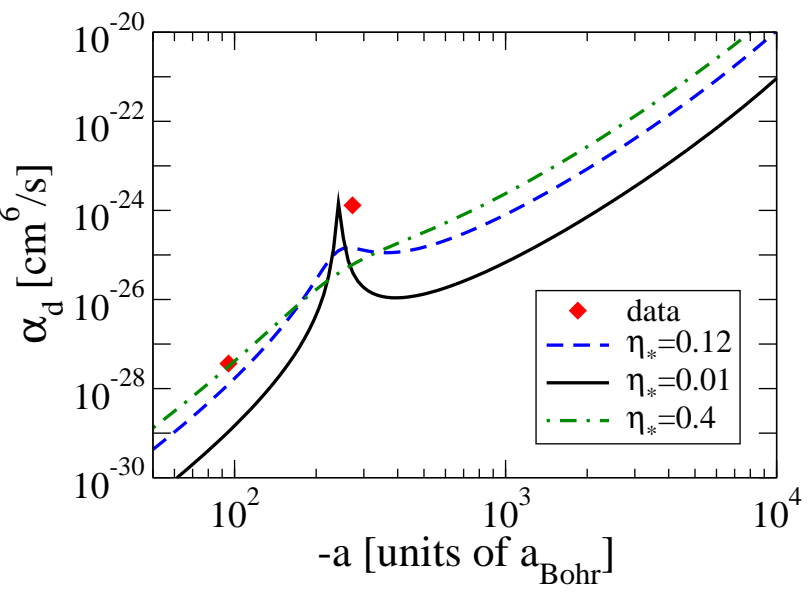

FIG. 8: (Color online) $\alpha_{d}$ vs $a$ for the ${ }^{41} \mathrm{~K}^{-87} \mathrm{Rb}_{-}{ }^{87} \mathrm{Rb}$ system assuming $a_{-}=-246 a_{\text {Bohr }}$ and three values of $\eta_{*}: \eta_{*}=0.12$ (dashed line), $\eta_{*}=0.01$ (solid line), and $\eta_{*}=0.4$ (dashdotted line). Data points indicated by diamonds are taken from Refs. [15, 42; ; see text.

Of course, the explicit numbers depend on the value of $\eta_{*}$, but one should not exclude the possibility of other explanations of the positive- $a$ feature.

Aside from detecting the positions of the resonances, it is desirable to compare the actual shapes of the $a$ dependence of the loss rate with the theoretical predictions, especially on the positive side of the resonance, where $\alpha_{s}+\alpha_{d}$ is rather smooth. So far, the three-body recombination rate in the ${ }^{87} \mathrm{Rb}-{ }^{41} \mathrm{~K}$ mixture has been measured for two values of $a<0$ [15, 43, and the comparison with our calculation is rather inconclusive. Figure 8 shows $\alpha_{d}$ for the K-Rb-Rb resonance at $a=-246 a_{B o h r}$ where only the recombination into deep dimers can occur. The dashed curve is calculated using the value $\eta_{*}=0.12$ suggested in Ref. 15. The data point close to the resonance is taken from Ref. [15, 43], whereas the one farther away from the resonance gives an upper limit of the recombination rate 42. In order to illustrate the sensitivity of the result to $\eta_{*}$, we also show it for $\eta_{*}=0.01$ (solid line) and $\eta_{*}=0.4$ (dash-dotted line). The discrepancy between the measured recombination rate at the resonance and our result for $\eta_{*}=0.12$ is about one order of magnitude. In order to understand its origin, more mea- surements around the resonance position are required. Such data would allow for a more precise determination of the width parameter $\eta_{*}$ and of the resonance shape predicted by the universal theory.

\section{Future experiments}

The Tübingen group of Zimmermann recently studied the ${ }^{7} \mathrm{Li}^{8}{ }^{8} \mathrm{Rb}$ and ${ }^{6} \mathrm{Li}^{8}{ }^{87} \mathrm{Rb}$ mixtures. They identified and quantified several interspecies Feshbach resonances in both of them [44, 45] and have reached quantum degeneracy [46. These mixtures are characterized by quite small mass ratios and, therefore, small scaling factors, which is favorable for observing the discrete scaling invariance. Another very good candidate for studying the Efimov effect with even smaller scaling factors is a mixture of ${ }^{133} \mathrm{Cs}$ with either isotope of lithium (the ${ }^{7} \mathrm{Li}^{133} \mathrm{Cs}$ mixture was created in Heidelberg [47). The universal parameters for these mixtures can be found in Table [. Predictions for the three-body recombination and atomdimer relaxation rates can be obtained from Eqs. (13), (16), 20), 23), and (25).

\section{SUMMARY AND CONCLUSION}

In this paper, we have calculated the three-body loss rates in heteronuclear mixtures of atoms for the case of large scattering length between the unlike atoms. We have analyzed this problem using two complementary methods.

First, we have formulated a universal effective field theory for this system and derived momentum-space integral equations for the trimer energies and the atom-dimer scattering amplitude. From an analysis of the boundstate equation we have calculated the ratio of the resonance positions $a_{*} /\left|a_{-}\right|$as a function of the mass ratio, $\delta$. Moreover, we have calculated the three-body recombination and atom-dimer relaxation rates numerically. We have provided semianalytical expressions for the rate constants of three-body recombination into shallow and deep dimers as a function of the interspecies scattering length $a$ and the Efimov width parameter $\eta_{*}$. Furthermore, we have calculated the atom-dimer relaxation constant from 
the scattering threshold at $E=-B_{d}$ up to the dimer breakup threshold at $E=0$.

Second, we have carried out an analysis of the recombination and relaxation process in configuration space. We have generalized the method developed in Ref. 36. to heteronuclear bosonic mixtures and obtained analytic expressions for the recombination and relaxation rates at $E=0$. We find excellent agreement of these expressions with our numerical results from the momentum-space integral equations.

The expressions in Eqs. (13), (16), (25), and (56) fully determine the three-body recombination rates for heteronuclear bosonic mixtures with resonant scattering between the unlike atoms in the universal zero-range theory. The atom-dimer relaxation rates at $E=-B_{d}$ and $E=0$ are given by Eqs. (18), (20), and (23). These equations are universal and can be used to analyze experimental data for any combination of atoms with the range applicability of the universal theory.

In Ref. [48, D'Incao and Esry give a general functional dependence of the recombination rates on the scattering length for all possible combinations of bosons and fermions. This includes the case of two identical bosons and a third atom with $J=0$ which we address here. We agree with their expressions for $\alpha_{d}$ in the case $a<0$ and for $\beta$. For $\alpha_{s}$, our general form (54) does not agree with their result. The proportionality of $\alpha_{s}$ to $\sin ^{2}\left(s_{0} \ln a+\phi_{3}\right)$ where $\phi_{3}$ is a short-range phase [48, 49 emerges only if $\exp \left(2 \pi s_{0}\right) \gg 1$ and the expression (54) can be simplified (cf. Ref. [1]). This is the case for small mass ratios $\delta$. Moreover, our prediction for the dependence of $\alpha_{s}$ on $\delta$ [see Eqs. (13) and (56)] differs from the result $\alpha_{s} \propto[\delta(2+\delta)]^{3 / 2} a^{4} /(1+\delta)^{2} / m_{1}$ obtained in Ref. [48.

We have applied our results to some heteronuclear mixtures in ongoing and planned experiments. We find good agreement between theory and the JILA experiment 28 . that investigated ${ }^{40} \mathrm{~K}-{ }^{8}{ }^{8} \mathrm{Rb}$ molecules and their stability in collisions with atoms near a wide heteronuclear Feshbach resonance at $B_{0}=546.7 \mathrm{G}$. For the recent experiment by the Florence group which uses a mixture of ${ }^{41} \mathrm{~K}$ and ${ }^{87} \mathrm{Rb}$ atoms [15, 43, we observe moderate discrepancies between theory and experiment. We obtain $a_{*} /\left|a_{-}\right|=0.52$ for the resonance positions while the ex- perimental ratio is $a_{*} /\left|a_{-}\right|=2.7$. Because neither the effective range corrections nor the experimental errors of the ratio are known accurately, no definite conclusion can be drawn at the moment. In particular, our analysis of atom-dimer relaxation suggests that explanations should be considered other than an Efimov resonance for the feature at $a=667 a_{B o h r}$ that was used to extract the value of $a_{*}$.

Using the value $\eta_{*}=0.12$ extracted in Ref. [15], we find that the calculated recombination rate at the resonance is about one order of magnitude too small. Using smaller values of $\eta_{*}$, the size of the experimental rate can be reproduced. In order to resolve this discrepancy, more measurements around the resonance position are required. Currently, there are only two data points and $\eta_{*}$ cannot be determined accurately. Additional data would allow for a more precise determination of $\eta_{*}$ and allow for a test of the resonance shape predicted by the universal theory.

Finally, we have calculated the universal parameters determining the three-body loss rates for various other mixtures and have summarized them in Table I. Extending earlier work by D'Incao and Esry [48, 49], our predictions lay the theoretical ground for the experimental observation of Efimov physics in heteronuclear mixtures. They should be useful for planning and analyzing future experiments.

\section{Acknowledgments}

We thank Eric Braaten, David Canham, and Francesco Minardi for discussions. K.H. was supported by the "Studienstiftung des Deutschen Volkes" and by the Bonn-Cologne Graduate School of Physics and Astronomy. H.W.H. acknowledges support from the the Bundesministerium für Bildung und Forschung, BMBF under Contract No. 06BN9006. D.S.P. is supported by the the Île-de-France Cold Atom Research Institute, IFRAF, by the French National Research Agency, ANR (Grant No. 08-BLAN-65), by the EuroQUAM-FerMix program, and by the Russian Foundation for Fundamental Research.
[1] E. Braaten and H.-W. Hammer, Phys. Rept. 428, 259 (2006) arXiv:cond-mat/0410417.

[2] L. Platter, Few Body Syst. 46, 139 (2009) arXiv:0904.2227.

[3] V. Efimov, Phys. Lett. 33B, 563 (1970).

[4] E. Nielsen and J.H. Macek, Phys. Rev. Lett. 83, 1566 (1999).

[5] B.D. Esry, C.H. Greene, and J.P. Burke, Phys. Rev. Lett. 83, 1751 (1999).

[6] P.F. Bedaque, E. Braaten, and H.-W. Hammer, Phys. Rev. Lett. 85, 908 (2000) arXiv:cond-mat/0002365.

[7] E. Braaten and H.-W. Hammer, Phys. Rev. Lett. 87,
160407 (2001) arXiv:cond-mat/0103331.

[8] E. Braaten and H.-W. Hammer, Phys. Rev. A 70, 042706 (2004) arXiv:cond-mat/0303249.

[9] T. Kraemer, M. Mark, P. Waldburger, J.G. Danzl, C. Chin, B. Engeser, A.D. Lange, K. Pilch, A. Jaakkola, H.-C. Nägerl, and R. Grimm, Nature 440, 315 (2006) arXiv:cond-mat/0512394.

[10] S. Knoop, F. Ferlaino, M. Mark, M. Berninger, H. Schoebel, H.-C. Naegerl, and R. Grimm, Nature Phys. 5, 227 (2009) arXiv:0807.3306.

[11] K. Helfrich and H.-W. Hammer, Europhys. Lett. 86, 53003 (2009) arXiv:0902.3410. 
[12] M. Zaccanti, B. Deissler, C. D'Errico, M. Fattori, M. Jona-Lasinio, S. Müller, G. Roati, M. Inguscio, and G. Modugno, Nature Phys. 5, 586 (2009) arXiv:0810.3876.

[13] N. Gross, Z. Shotan, S. Kokkelmans, and L. Khaykovich, Phys. Rev. Lett. 103, 163202 (2009) arXiv:0906.4731.

[14] S.E. Pollack, D. Dries, and R.G. Hulet, arXiv:0911.0893

[15] G. Barontini, C. Weber, F. Rabatti, J. Catani, G. Thalhammer, M. Inguscio, and F. Minardi, Phys. Rev. Lett. 103, 043201 (2009) arXiv:0901.4584.

[16] T. B. Ottenstein, T. Lompe, M. Kohnen, A. N. Wenz, and S. Jochim, Phys. Rev. Lett. 101, 203202 (2008) arXiv:0806.0587.

[17] J.H. Huckans, J.R. Williams, E.L. Hazlett, R.W. Stites, and K.M. O'Hara, Phys. Rev. Lett. 102, 165302 (2009) arXiv:0810.3288.

[18] E. Braaten, H.-W. Hammer, D. Kang and L. Platter, Phys. Rev. Lett. 103, 073202 (2009) arXiv:0811.3578.

[19] P. Naidon and M. Ueda, Phys. Rev. Lett. 103, 073203 (2009) arXiv:0811.4086.

[20] S. Floerchinger, R. Schmidt, and C. Wetterich, Phys. Rev. A 79, 053633 (2009) arXiv:0812.1191.

[21] A.N. Wenz, T. Lompe, T.B. Ottenstein, F. Serwane, G. Zürn, and S. Jochim, Phys. Rev. A 80, 040702(R) (2009) arXiv:0906.4378.

[22] J.R. Williams, E.L. Hazlett, J.H. Huckans, R.W. Stites, Y. Zhang, and K.M. O'Hara, Phys. Rev. Lett. 103, 130404 (2009) arXiv:0908.0789.

[23] S. Jochim (private communication).

[24] E. Braaten, H.-W. Hammer, D. Kang and L. Platter, Phys. Rev. A 81, 013605 (2010) arXiv:0908.4046.

[25] R.D. Amado and J.V. Noble, Phys. Rev. D 5, 1992 (1972).

[26] V. Efimov, JETP Lett. 16, 34 (1972)

[27] V. Efimov, Nucl. Phys. A 210, 157 (1973).

[28] J.J. Zirbel, K.-K. Ni, S. Ospelkaus, J.P. D'Incao, C.E. Wieman, J. Ye, and D.S. Jin, Phys. Rev. Lett. 100, 143201 (2008) arXiv:0710.2479.

[29] P.F. Bedaque, H.-W. Hammer, and U. van Kolck, Phys. Rev. Lett. 82, 463 (1999) arXiv:nucl-th/9809025; Nucl. Phys. A 646, 444 (1999) arXiv:nucl-th/9811046.

[30] H.-W. Hammer and T. Mehen, Nucl. Phys. A 690, 535 (2001) arXiv:nucl-th/0011024.

[31] M.A. Efremov, L. Plimak, B. Berg, M.Yu. Ivanov, and W.P. Schleich, Phys. Rev. A 80, 022714 (2009) arXiv:0905.3974.

[32] J.H. Macek, S. Ovchinnikov, and G. Gasaneo, Phys. Rev. A 72, 032709 (2005).
[33] J.H. Macek, S.Y. Ovchinnikov, and G. Gasaneo, Phys. Rev. A 73, 032704 (2006).

[34] D. S. Petrov, talk at the Workshop on Strongly Interacting Quantum Gases, Ohio State University, April 2005.

[35] A.O. Gogolin, C. Mora, and R. Egger, Phys. Rev. Lett. 100, 140404 (2008) arXiv:0802.0549.

[36] D. S. Petrov, Phys. Rev. A 67, 010703(R) (2003) arXiv:cond-mat/0209246.

[37] D. S. Petrov, C. Salomon, and G. V. Shlyapnikov, Phys. Rev. A 71, 012708 (2005) arXiv:cond-mat/0407579.

[38] In momentum space we have to work with an integral operator, the kernel of which is given by Eq. (5) with $E=0$. This operator possesses a property similar to Eq. 39, but the region of convergence in that case is narrower.

[39] L. D. Landau and E. M. Lifshitz, Quantum Mechanics (Butterworth-Heinemann, Oxford, 1997).

[40] Note that the coefficient $C(\delta)$ has the same dependence on $\delta$ as the term proportional to $\ln p$ in the definition of the infrared subtracted amplitude $\overline{\mathcal{A}}$ in Eq. 115 . This term arises from two-loop diagrams at fourth order in the two-body coupling $g_{2}$ (see Sec. 8.5 in Ref. [1] and references therein).

[41] Note that our rate constant $\alpha$ is half the three-body loss coefficient $K_{3}$ defined in Ref. [28].

[42] F. Minardi, private communication.

[43] G. Barontini, C. Weber, F. Rabatti, J. Catani, G. Thalhammer, M. Inguscio, and F. Minardi, Phys. Rev. Lett. 104, 059901(E) (2010).

[44] C. Marzok, B. Deh, C. Zimmermann, Ph.W. Courteille, E. Tiemann, Y.V. Vanne, and A. Saenz, Phys. Rev. A 79, 012717 (2009) arXiv:0808.3967.

[45] B. Deh, C. Marzok, C. Zimmermann, and Ph.W. Courteille, Phys. Rev. A 77, 010701(R) (2008) arXiv:0709.4554.

[46] C. Silber, S. Günther, C. Marzok, B. Deh, Ph.W. Courteille, and C. Zimmermann, Phys. Rev. Lett. 95, 170408 (2005) arXiv:cond-mat/0506217.

[47] J. Deiglmayr, A. Grochola, M. Repp, K. Mörtlbauer, C. Glück, J. Lange, O. Dulieu, R. Wester, and M. Weidemüller, Phys. Rev. Lett. 101, 133004 (2008) arXiv:0807.3272.

[48] J.P. D'Incao and B.D. Esry, Phys. Rev. A 73, 030702(R) (2006) arXiv:physics/0508119.

[49] J.P. D'Incao and B.D. Esry, Phys. Rev. A 73, 030703(R) (2006) arXiv:cond-mat/0508474. 